\title{
Cerebral and Lower Limb Near-Infrared Spectroscopy in Adults on Extracorporeal Membrane Oxygenation
}

\author{
Joshua K Wong \\ Thomas Jefferson University \\ Thomas N Smith \\ Thomas Jefferson University \\ Harrison T Pitcher \\ Thomas Jefferson University \\ Hitoshi Hirose \\ Thomas Jefferson University \\ Nicholas C. Cavarocchi \\ Thomas Jefferson University \\ Follow this and additional works at: https://jdc.jefferson.edu/surgeryfp \\ Part of the Surgery Commons \\ Let us know how access to this document benefits you
}

\section{Recommended Citation}

Wong, Joshua K; Smith, Thomas N; Pitcher, Harrison T; Hirose, Hitoshi; and Cavarocchi, Nicholas C., "Cerebral and Lower Limb Near-Infrared Spectroscopy in Adults on Extracorporeal Membrane Oxygenation" (2012). Department of Surgery Faculty Papers. Paper 76.

https://jdc.jefferson.edu/surgeryfp/76

This Article is brought to you for free and open access by the Jefferson Digital Commons. The Jefferson Digital Commons is a service of Thomas Jefferson University's Center for Teaching and Learning (CTL). The Commons is a showcase for Jefferson books and journals, peer-reviewed scholarly publications, unique historical collections from the University archives, and teaching tools. The Jefferson Digital Commons allows researchers and interested readers anywhere in the world to learn about and keep up to date with Jefferson scholarship. This article has been accepted for inclusion in Department of Surgery Faculty Papers by an authorized administrator of the Jefferson Digital Commons. For more information, please contact: JeffersonDigitalCommons@jefferson.edu. 
As submitted to:

Artificial Organs

And later published as:

Cerebral and Lower Limb Near-Infrared Spectroscopy in Adults on Extracorporeal Membrane Oxygenation (ECMO)

\author{
Volume 36, Issue 8, Pages 659-667, August 2012.
}

DOI: $10.1111 / \mathrm{j} .1525-1594.2012 .01496 . x$

Joshua K Wong BS, Thomas N Smith BS, Harrison Pitcher MD, Hitoshi Hirose MD, Nicholas C Cavarocchi MD

Division of Cardiothoracic Surgery, Department of Surgery

Thomas Jefferson University, Philadelphia, PA

Correspondence:

Nicholas C Cavarocchi, MD

Associate Professor of Surgery

Division of Cardiothoracic Surgery and Critical Care

Director, Surgical Cardiac Care Unit

Department of Surgery, Thomas Jefferson University

1025 Walnut Street, College Bldg, Suite 605E, Philadelphia, PA 19107

Phone: (215) 955-6996, Fax: (215) 923-6010

Email: Nicholas.Cavarocchi@jefferson.edu

Presented in part at the 19th Congress of the International Society for Rotary Blood Pumps, held September 8-10, 2011 in Louisville, Kentucky, USA. 


\begin{abstract}
Introduction: Percutaneous femoral Veno-arterial (VA) or jugular Veno-venous (VV) ECMO can result in delivery of hypoxic blood to the brain, coronaries and upper extremities. Additionally, VA-ECMO by percutaneous femoral artery cannulation may compromise perfusion to the lower limbs. Use of Near-Infrared Spectroscopy (NIRS) detects regional ischemia and warns of impending hypoxic damage. We report the first known series with standardized monitoring of this parameter in adults on ECMO.
\end{abstract}

Methods: This is an IRB approved single institution retrospective review of patients with NIRS monitoring on ECMO from July 2010 till June 2011. Patients were analyzed for drops in NIRS tracings below 40 or $>25 \%$ from baseline. VA-ECMO and VV-ECMO were initiated by cannulation of the femoral vessels and the internal jugular vein respectively. Sensors were | placed on the patients' foreheads and on the lower limbs. NIRS tracings were recorded, analyzed and correlated with clinical events

Results: Twenty patients were analyzed (Median age: 47.5 years), 17 patients were placed on VA-ECMO, and 3 patients on VV-ECMO. The median duration on ECMO was 7 days (Range 2 -26). $100 \%$ of patients had a significant drop in bilateral cerebral oximetry tracings resulting in hemodynamic interventions, which involved increasing pressure, oxygenation and/or ECMO flow. In 16 (80\%) patients, interventions corrected the underlying ischemia. 4 (20\%) patients required further diagnostic intervention for persistent decreased bilateral and/or unilateral cerebral oximetry tracings, and were found to have a cerebrovascular accident (CVA). Six | (30\%) patients had persistent unilateral lower limb oximetry events, which resolved upon placement or replacement of a distal perfusion cannula. No patient was found to have either lower limb ischemia or a CVA with normal NIRS tracings.

Conclusions: Use of NIRS with ECMO is important in detecting ischemic peripheral vascular and cerebral events. This allows for potential correction of the underlying process, thus preventing permanent ischemic damage.

Keywords: Extracorporeal Membrane Oxygenation, Adults, Near-Infrared Spectroscopy, Ischemia, Cerebral Oximetry, Extremity Oximetry 


\section{INTRODUCTION}

Extracorporeal Membrane Oxygenation also commonly referred to as ECMO, is a form of extracorporeal life support by a modified heart-lung machine that is potentially life saving in patients with cardio-respiratory failure. Its use was first described by Hill et al. in 1972, in the treatment of a young man with acute respiratory distress syndrome (ARDS) after a motor vehicle accident, ${ }^{1}$ and has since been increasingly used to temporarily support patients with cardiac, respiratory or combined cardio-respiratory failure for a period of days to weeks.

Traditionally the use of this form of support was reserved primarily for the pediatric population, especially in the treatment of neonatal respiratory failure, ${ }^{2}$ but its initial application in adults was limited. Early trials in adults were disappointing, with poor outcomes and no survival benefit being reported over conventional treatment. ${ }^{3,4}$ However, with advancements in mechanical cardio-respiratory technology, such as the development and use of new and improved centrifugal pumps and oxygenators, ${ }^{5-7}$ and increasing center experience in the management of these patients, outcomes for adults that are placed on this form of support are improving, and the use of ECMO in this population is increasing. ${ }^{8-10}$

ECMO can be divided into 2 basic types. Veno-Arterial or VA-ECMO is used to support both the heart and lungs. Common indications for VA-ECMO are; failure to wean from cardiopulmonary bypass (CPB), cardiogenic shock secondary to multiple etiologies as a bridge to device or transplant for stabilization and/or recovery of end organs, etc. Veno-Venous or VVECMO is used for lung support alone and is used primarily in patients with potentially reversible respiratory failure, such as ARDS, that is refractory to conventional management. ${ }^{4,10}$

It is important to note that the successful use of ECMO is determined not only by technical factors related to circuit design or clinician experience, but also on the various monitoring systems that are employed by clinicians to evaluate the critically ill patient. NearInfrared Spectroscopy (NIRS) is one such monitoring system. It employs the use of nearinfrared wavelengths emitted by sensor pads to evaluate regional oxygenation of the organ or tissue being monitored. The difference in absorption of these wavelengths by oxygenated and deoxygenated hemoglobin is calculated, providing the clinician with information regarding the regional oxygen saturation $\left(\mathrm{rSO}_{2}\right)$ of the perfused tissue.

The use of NIRS is well described in the literature for use in patients undergoing cardiopulmonary bypass in cardiac surgery. The application of this technology in cardiac surgery patients has proven beneficial in many major studies, ${ }^{11-12}$ however there is yet to be a report in the English literature of its use in a series of adult patients on ECMO for the purpose of monitoring cerebral and femoral perfusion. In this report, we provide a single institution experience with the use of cerebral and lower limb NIRS in Adults on ECMO.

\section{METHODS}

We performed an Institutional Review Board_(IRB) approved, review of all adult patients that were placed on ECMO with NIRS monitoring from July 2010 to June 2011 in the Surgical Cardiac Care Unit of Thomas Jefferson University Hospital. Patient data was reviewed retrospectively from a prospective data collecting system. Patient demographics, indications for ECMO, duration on ECMO, occurrence of cerebral or extremity events, and neurologic or lower limb complications were analyzed and reported.

Neurological injury was defined as the occurrence of a hemorrhagic and/or ischemic infarct and/or diffuse anoxic brain injury, that was confirmed by neuro-imaging (MRI, CT Scan, Nuclear brain perfusion scan, EEG) or in the case of brain death serial exams 24-hours apart by 
two independent neurologists. Lower limb complications were classified into two categories; "need for fasciotomy" and "loss of limb".

VA-ECMO/VV-ECMO Cannulation Method: All twenty patients were cannulated peripherally via percutaneous insertion of the ECMO cannula using the Seldinger technique. Femoral cannulation was the preferred placement in all patients on VA-ECMO. All patients on VV-ECMO were cannulated via the neck into the internal jugular vein using the Avalon ${ }^{\mathrm{TM}}$ bicaval dual-lumen cannula. Distal arterial perfusion ports were placed in all patients with peripheral femoral cannulation.

The heart and lung support (HLS) Maquet cannulae (bio-compatible polyurethane) from the patient's blood vessels were connected to a closed crystalloid primed circuit $(\sim 300 \mathrm{ml})$; both cannulae and tubing have heparin coating. The circuitry for ECMO consisted of the Quadrox-D (diffusion membrane hollow-fiber) oxygenator and a Rota flow centrifugal pump (Maquet Cardiovascular LLC, San Jose, California) with a heater or cooler exchange.

NIRS Device: $\quad$ The INVOS ${ }^{\mathrm{TM}}$ cerebral somatic oximeter sensor pads were placed bilaterally on the forehead (Figure 1a) in all patients, and medially on the left and right lower

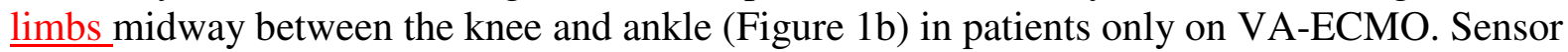
pads were replaced every 72 hours, or earlier if indicated. Values that represented the adequacy of tissue oxygenation were calculated by the device and assigned values called " $\mathrm{SS}_{2}$ " (Regional Oxygen Saturation). A 4-channel monitor screen placed at the bedside of every patient, presented a 4-hour view of the cerebral and lower limb tracings (Figure 1c), which allowed the clinician to track the oxygenation of cerebral and distal limb tissues over time. Monitoring of this parameter was initiated within minutes upon placement on ECMO, and was discontinued only after a successful wean to recovery, bridge to a ventricular assist device or death.

Baseline values were established early in the course of ECMO therapy and $\mathrm{rSO}_{2}$ numbers were compared to the previously established baseline daily. Clinically significant events warranting interventions were defined as below;

1) Drop in $\mathrm{rSO}_{2}$ values below 40.

2) Drop in $\mathrm{rSO}_{2}$ values more than $25 \%$ from baseline.

A bilateral drop in cerebral tracings that responded to interventions was termed a "Systemic Event", a bilateral or unilateral drop in cerebral tracings that was secondary to neurological injury was termed a "Cerebral Event" and a unilateral drop in extremity tracings that required an intervention was termed a "Lower Limb Event". Tracings were recorded, analyzed and correlated with clinical events.

ECMO Cerebral Oximetry Management: Management protocols for intervention were established at the beginning of the study period for patients who had clinically significant drops in cerebral $\mathrm{rSO}_{2}$ values. All patients who had drops in either bilateral or unilateral cerebral $\mathrm{rSO}_{2}$ values were managed first by ruling out mechanical causes (head position, proper placement of ECMO cannulae, and sensor pad placement). Should mechanical causes be ruled out, our next step in management involved increasing the oxygen and/or blood supply to tissues by increasing ECMO flow, ECMO oxygen supply, mean arterial pressure and ensuring normal hemoglobin levels. If the interventions delineated above did not resolve the low values, patients with persistent low bilateral $\mathrm{rSO}_{2}$ readings were placed on the "Persistent Bilateral Decreased Cerebral Tracing Protocol" (Figure 2a), whereas patients with persistently low unilateral $\mathrm{rSO}_{2}$ readings went directly to neuro-imaging (Figure $2 b$ ).

ECMO Lower Limb Oximetry Management: Similar management protocols were established for patients on VA-ECMO with clinically significant drops in lower limb oximetry 
values. Patients with significant drops in $\mathrm{rSO}_{2}$ values on the side of arterial cannulation were started on the "Decreased Lower Limb Tracing Protocol" (Figure 2c). Interventions invariably led to a restoration of previously established baseline readings or prophylactic fasciotomy to prevent the development of compartment syndrome and subsequent limb loss.

VV-ECMO Oximetry Management: The application of NIRS in patients on VV-ECMO is similar to its use in patients on VA-ECMO. It is used to primarily monitor for neurological injury. However, our unit also relied on NIRS in conjunction with Chest X-Ray's, Arterial Blood Gas values and Pulse oximetry in guiding our weaning protocol. Patients were weaned off VVECMO if all the following criteria were met; NIRS tracings were similar to or $>$ baseline readings, Pulse oximeter readings $>85 \%$, Minimal ventilator settings, ECMO FiO 2 at $50 \%$ or less, and a clear or markedly improved chest x-ray.

\section{RESULTS}

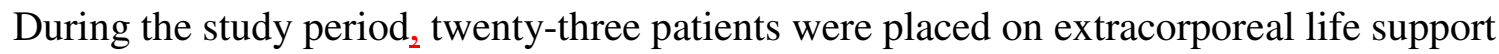
via ECMO. A total of twenty patients had cerebral and/or lower limb oximetry monitoring using NIRS technology. Of these twenty, eleven $(55 \%)$ were male. The median age of our patient population was 47.5 years (Range 17 - 74) and the median duration on ECMO was 7 days (Range 2 - 26). Seventeen (85\%) patients were supported by VA-ECMO and the remaining three $(15 \%)$ by VV-ECMO. The main and only indication for placement on VV-ECMO was ARDS $(3 / 3,100 \%)$. The two most common indications for initiation of VA-ECMO were; Failure to wean from CPB $(4 / 20,20 \%)$ and Cardiogenic shock secondary to Acute Myocardial Infarction (4/20, 20\%). The remaining indications for placement on VA-ECMO are as described in Table 1.

Upon analysis of tracings and correlation with clinical events, it was noted that all twenty $(100 \%)$ patients on ECMO had a bilateral drop in cerebral tracings. Interventions as outlined above were undertaken to resolve these low readings, which succeeded in sixteen patients $(16 / 20,80 \%)$ (Systemic Event, Table 2). An example of this is shown in Figure 3a and Figure 3b. Of the four that did not respond to interventions (Cerebral Event), two patients had persistent bilateral low $\mathrm{rSO}_{2}$ readings, and were eventually sent for neuro-imaging as outlined in the | "Persistent Bilateral Decreased Cerebral Tracing Management Protocol", which confirmed diffuse anoxic brain injury in both patients. Following serial neurological exams 24-hours apart by neurologists, they were declared brain dead. The remaining two patients had persistent unilaterally low $\mathrm{rSO}_{2}$ readings, and were sent directly for neuro-imaging that revealed large unilateral infarcts. These events are summarized in Table 2 and the cerebral tracing graph demonstrating this event is shown in Figure 4.

Of the seventeen patients that were placed on VA-ECMO, six $(6 / 17,35 \%)$ had a clinically significant drop in unilateral lower limb tracings (Lower Limb Event). Interventions as outlined above in the "Decreased Lower Limb Tracing Protocol" were undertaken, which resolved the low $\mathrm{rSO}_{2}$ readings in all six patients $(6 / 6,100 \%)$. However, four patients $(4 / 6,67 \%)$ required prophylactic distal limb two-compartment fasciotomies against compartment syndrome. There was no loss of limbs in patients peripherally cannulated via the femoral vessels in our study (Table 3). Figure 5 depicts the development of a "Lower Limb Event" in a patient monitored by NIRS technology. Lastly, no patient with normal cerebral or lower limb tracings was found to have neurological injury or lower limb ischemia.

In the three patients that were placed on VV-ECMO in our study, two were successfully weaned off ECMO using the criteria as mentioned above. NIRS along with other factors guided the weaning of these patients from ECMO and contributed to their successful discharge and 
overall survival. However, one patient had a large stroke that was detected by cerebral oximetry and due to her poor prognosis care was withdrawn.

\section{DISCUSSION}

The potential application of NIRS technology in patients was observed as early as 1977 by Jobsis et al. in a report that described the possibility of real time noninvasive monitoring of tissue oxygen saturation using trans-illumination spectroscopy which was possible due to the high degree of transparency of myocardial and brain tissue in the near-infrared (NIR) range. ${ }^{13}$ However, it was not until 1985 that Ferrari et al. studied the application of this technology (NIRS) in human patients, and was among the first to report its use and application in a clinical setting. ${ }^{14}$

As stated above, the use and application of NIRS in patients is well studied, particularly in the cardiac surgical population. In a retrospective study by Goldman et al. in 2004, 1034 patients (study group) who underwent cerebral oximetry monitoring using NIRS technology during cardiac surgery were compared to 1245 patients (control group) who were not monitored by this oxygenation parameter. It was found that patients in the study group had fewer permanent strokes, and shorter hospital stays. ${ }^{11}$ Murkin et al. published further evidence to its utility in 2007 in an important prospective randomized trial. In his report, 100 patients that were stratified to intra-operative cerebral regional oxygen saturation $\left(\mathrm{rSO}_{2}\right)$ monitoring with active treatment of declining values demonstrated shorter intensive care unit duration stays, and lower major organ morbidity or mortality, when compared to a group of patients that underwent blinded $\mathrm{rSO}_{2}$ monitoring. ${ }^{12}$ The reliability of this device in detecting neurological events has also been well reported in the literature. Samra et al reported a sensitivity of $80 \%$ and a specificity of $82.2 \%$ in detecting strokes at a NIRS tracing threshold of $>20 \%$ change from baseline, ${ }^{15}$ whereas Ali et al reported a sensitivity of $75 \%$ and a specificity of $97.5 \%$ at a similar threshold in patients undergoing carotid endarterectomies. ${ }^{16}$

To the best of our knowledge, there are only two mentioned reports of NIRS use in patients on ECMO, and all of them in neonates or children. ${ }^{17}, 1 \underline{8}$ Papademetriou et al. described the monitoring of cerebral and tissue oxygenation of 4 children on ECMO as they underwent manipulations in circuit flow. The aim of his study was to compare variation in $\mathrm{rSO}_{2}$ values at different near-infrared light frequencies ${ }^{17}$ whereas Rais-Bahrami et al. in another study attempted to validate NIRS value accuracy by periodically obtaining cephalic blood samples and comparing it to $\mathrm{rSO}_{2}$ numbers in 17 neonates. ${ }^{18}$ No study to date has described its use, application and utility in a clinical setting in a series of adult ECMO patients.

A question could then be posed as to why NIRS monitoring is beneficial in patients on ECMO? The authors believe that it is so for the following reasons. Firstly, in a peripherally cannulated ECMO patient, oxygenated blood flows in a retrograde fashion, travelling proximally towards the heart. This flow is opposed by potentially poorly oxygenated or deoxygenated blood (dependent on respiratory status) pumped by a still beating, although weakened heart. With high ECMO flow and a low pulse pressure, the mixing oxygenated and poorly oxygenated (or deoxygenated) blood occurs at a level proximal to the bifurcation of the great vessels allowing for the provision of oxygenated blood to the brain and upper extremities (Figure 6 a). However, with increasing pulse pressures or increased resistance to ECMO flow, this level of mixing can potentially occur distal to the bifurcation of these vessels rendering these organs susceptible to ischemic damage ${ }^{5}$ (Figure $6 \mathrm{~b}$ ). The level at which this mixing occurs can be hard to define with no good technique reported to date. However, the side effects of mixing distal to the great vessels 
can be evaluated by cerebral oximetry, which potentially prevents the development of permanent ischemic damage to an already vulnerable brain.

Secondly, a major complication that can occur in patients supported by ECMO is the development of neurological injury. Studies have shown that neurological complications are greatly underappreciated and occur with relative frequency in ECMO patients. A recent retrospective review by Mateen et al. noted that of 84 patients that underwent ECMO at the Mayo Clinic, $42(50 \%)$ patients developed some form of neurological injury. ${ }^{19}$ This rate may seem relatively high compared to other similar studies, but it is noteworthy that the authors definition of neurological injury was broader and included categories such as "encephalopathy, myoclonus, and comatose without further explanation". Most other studies report only the rates of ischemic or hemorrhagic strokes, and that ranges from $7 \%-21 \%, \underline{20}, \underline{21}$ This however represents a still high number and warrants concern in any ECMO center. In our experience, neurological complications occurred in $20 \%$ of our patients, which is in accordance with the rates reported in the literature. It emphasizes the importance of having a clinically validated neuro-monitoring system that can detect developing ischemic damage with the hope of potentially reversing it. Furthermore, the ability of the clinician to know objectively that the brain is well perfused is an essential piece of information as it can be difficult to determine the true neurological status of an intubated, often paralyzed and heavily sedated patient.

Lastly, in VA-ECMO patients with peripheral femoral artery cannulation, distal limb ischemia represents a potentially hazardous complication. This is due to the large cannula size relative to the diameter of the femoral artery that near occludes the vessel with resultant downstream ischemia. In our experience, placement of a distal perfusion cannula into a vessel distal to the site of ECMO cannulation ensures that there is downstream flow and lower limb oximetry (NIRS) monitors the adequacy of that flow. A report of distal limb ischemic complications in ECMO patients observed a complication rate of $21 \%$ in patients who did not receive a distal perfusion cannula, and no distal limb complications in patients who had one placed. ${ }^{22}$ In our group of 17 patients, distal perfusion cannulae were placed prophylactically but femoral events still occurred that warranted intervention, highlighting the still likely possibility of developing distal limb ischemic damage with a distal perfusion cannula. Clinical assessment of compartment syndrome has always been subjective and unreliable especially in critically ill patients that are incapable of communicating pain or sensation. The only reliable method to diagnose and prevent the development of this syndrome in such situations (manual measurement of compartment pressure) is invasive, which requires the use of hollow bore needles attached to pressure transducers that are inserted into muscle compartments. However, recent literature suggests that ischemia and decreased blood flow may increase muscle damage at lower compartment pressures making the diagnosis of this syndrome inherently more difficult and the use of pressure transducers less effective. ${ }^{23}$ In 1999, Arbabi et al studied the ability of NIRS to detect compartment syndrome in conditions of severe shock by firstly reducing mean arterial pressure and oxygen delivery (induce shock), and subsequently infusing colloidal albumin into the muscle compartments of pigs (induce compartment syndrome). They found that there was a significant difference in NIRS values in pigs prior to infusion of albumin compared to values after the development of compartment syndrome. Additionally, they noted that there was a significant difference in NIRS values in pigs that developed compartment syndrome compared to a control group of pigs in which compartment syndrome was not induced. ${ }^{23}$ Recently the same group then directly compared the utility of NIRS against compartment pressure in diagnosing compartment syndrome in human volunteer subjects. They found that NIRS had a higher 
sensitivity when compared with compartment pressure at the same specificity. ${ }^{24}$ In summary, NIRS is a better modality to monitor for compartment tissue ischemia due to its non-invasive nature and may even prove to be better at diagnosing this syndrome because it is a direct measure of tissue ischemia and also due to its ability to integrate the multiple factors that contribute to the development of compartment syndrome.

\section{LIMITATIONS}

Despite its obvious benefits, NIRS technology is not without any limitations. Its absolute values can be quite inaccurate and there exists significant variation in readings between patients. This potentially makes it difficult for the clinician to interpret the actual numbered readings but we emphasize the importance of establishing a baseline, in order to better understand any subsequent reading.

NIRS values can also be affected by extraneous factors such as ambient light and skin color (correlates with the amount of melanin in the skin), however our experience with use of this technology has posed no difficulty in minimizing or compensating for these factors.

\section{CONCLUSION}

We conclude this report with a reiteration on the importance of monitoring tissue oxygenation in patients on ECMO, particularly in the brain and distal limb. We have found that NIRS technology is a useful and highly applicable monitoring parameter that not only provides us with important bedside "point of care" information, but also serves as a new "vital sign" that has improved the quality of care and outcomes of critically ill ECMO patients in the surgical cardiac care unit at our institution. 
Table 1. Indications for extracorporeal membrane oxygenation (ECMO)

\begin{tabular}{|l|c|}
\hline Indications & Number $(n=20)$ \\
\hline Cardiac arrest or cardiogenic shock & $7(35 \%)$ \\
Post-cardiotomy shock or failure to wean off cardiopulmonary bypass & $4(20 \%)$ \\
Acute respiratory distress syndrome & $5(25 \%)$ \\
Myocarditis & $2(10 \%)$ \\
Decompensated heart failure & $1(5 \%)$ \\
Rejection of heart transplant & $1(5 \%)$ \\
\hline
\end{tabular}

Table 2. Summary of systemic and cerebral events

\begin{tabular}{|l|c|}
\hline Patients on ECMO $(V A-$ and $V V$-) & Number $(n=20)$ \\
\hline Bilateral drop in cerebral tracings (Systemic event) & $20(100 \%)$ \\
Resolved with interventions & $16(80 \%)$ \\
\hline Persistent bilateral/unilateral drop in cerebral tracings (Cerebral event) & $4(20 \%)$ \\
Confirmed neurological injury & $4(20 \%)$ \\
\hline
\end{tabular}

Table 3. Summary of lower limb events

\begin{tabular}{|l|c|}
\hline Patients on VA-ECMO & Number $(n=17)$ \\
\hline Unilateral drop in extremity tracings (Lower limb event) & $6(35 \%)$ \\
Resolved with interventions & $6(35 \%)$ \\
\hline Need for fasciotomy & $4(24 \%)$ \\
\hline Loss of limb & $0(0 \%)$ \\
\hline
\end{tabular}




\section{Legends of figures}

Figure 1: 1a (top). Left and right INVOS ${ }^{\text {TM }}$ cerebral somatic oximeter sensor pads placed on the forehead of the patient

$1 \mathrm{~b}$ (middle). INVOS ${ }^{\mathrm{TM}}$ cerebral somatic oximeter sensor pad placed on lower limb (mid calf).

1c (bottom). INVOS ${ }^{\mathrm{TM}}$ cerebral somatic oximeter 4-channel monitor screen showing left cerebral regional oxygen saturation, $\mathrm{rSO}_{2}=48$, right cerebral $\mathrm{rSO}_{2}=58$, left leg $=$ $\mathrm{rSO}_{2} 69$, and right leg $=\mathrm{rSO}_{2} 47$ (bottom).
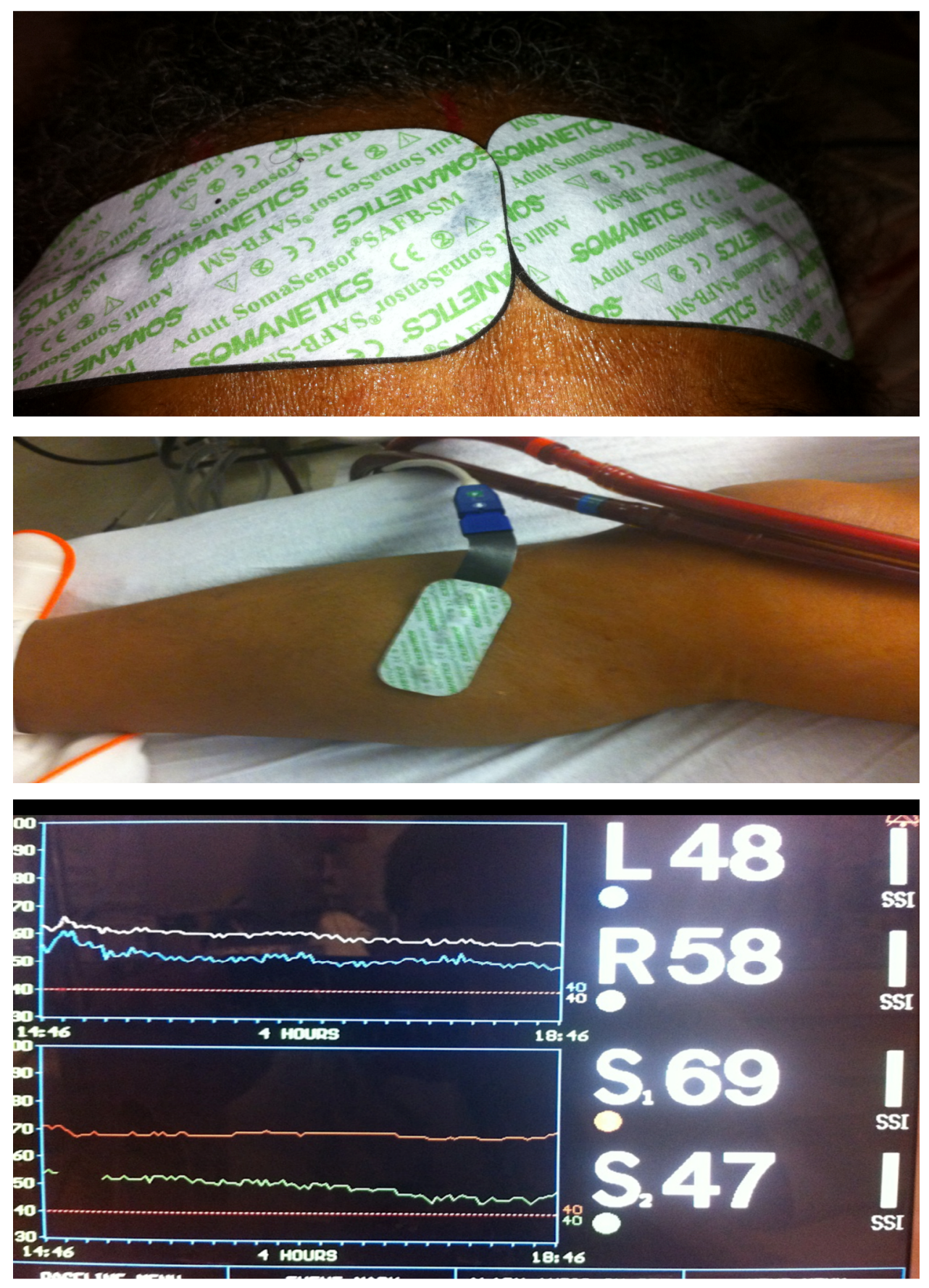
Figure 2: $2 \mathrm{a}$ (Left). Treatment protocol for persistent unilateral decreased cerebral tracing $\underline{\text { s }}$. $2 \mathrm{~b}$ (Middle). Treatment protocol for persistent bilateral decreased cerebral tracings. 2c (Right). Treatment protocol for persistent decreased lower limb tracings.

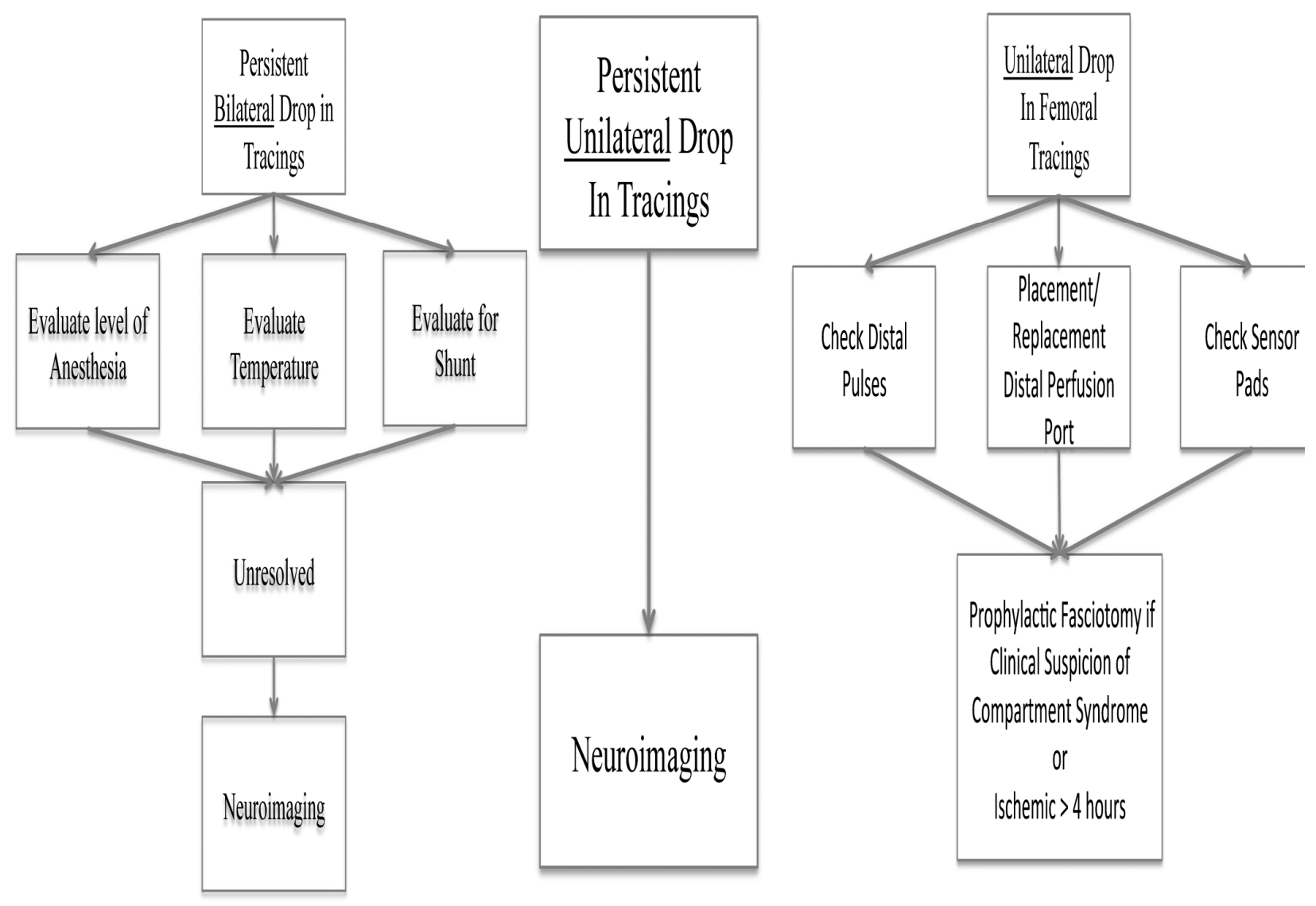


Figure 3. 3a (Top). Baseline tracing of a patient on ECMO (top). Blue line indicates trend of the right cerebral regional oxygen saturation $\left(\mathrm{rSO}_{2}\right)$, gray line indicates trend of the left cerebral $\mathrm{rSO}_{2}$, orange line indicates trend of the right lower limb $\mathrm{rSO}_{2}$, and green line indicates trend of the left lower limb $\mathrm{rSO}_{2}$.

$3 \mathrm{~b}$ (Bottom). Development of a systemic event noted by INVOS $\stackrel{\text { TM }}{\text {. Decreased bilateral }}$ cerebral and lower limb rSO 2 tracings was observed and returned to baseline by blood transfusion.

\section{$\underline{\text { Baseline Tracings }}$}

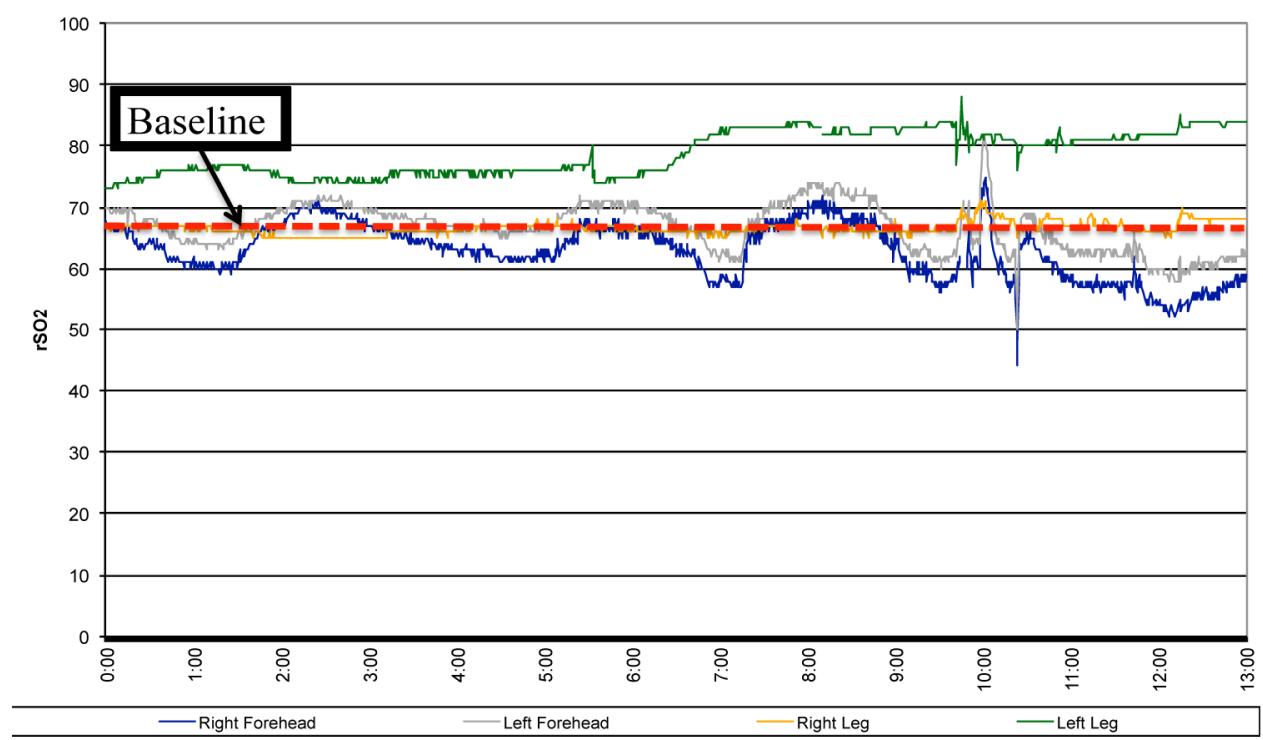

\section{$\underline{\text { Systemic Event }}$}

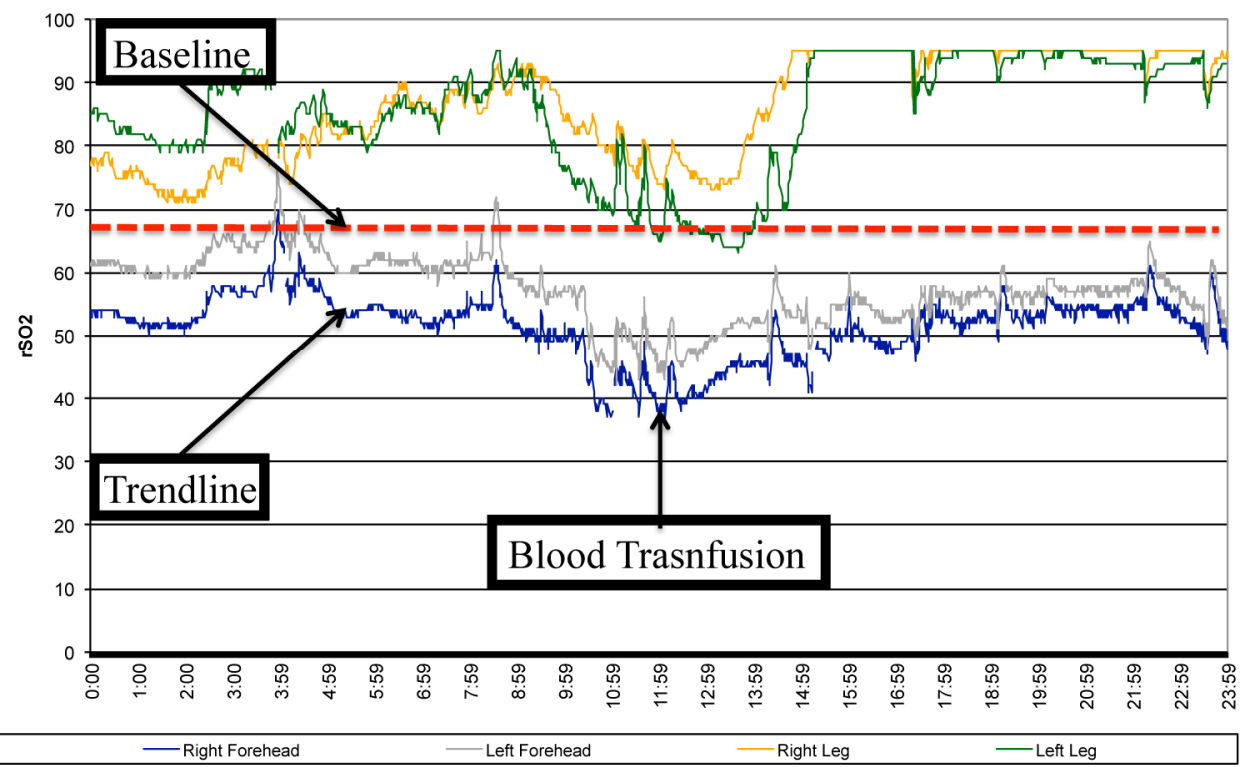


Figure 4. A cerebral event noted by NIRS. Day 1: Normal bilateral cerebral rSO2 tracings. Day 2: Left cerebral rSO2 tracing drop indicating early perfusion decompensation. Day 4: Further investigation (neuro-imaging) of persistently low cerebral rSO2 diagnosed a left middle cerebral artery infarct. Day 5: Subsequent drop in right cerebral rSO2 secondary to severe cerebral edema and loss of bilateral cerebral blood flow.

\section{$\underline{\text { Cerebral Event }}$}

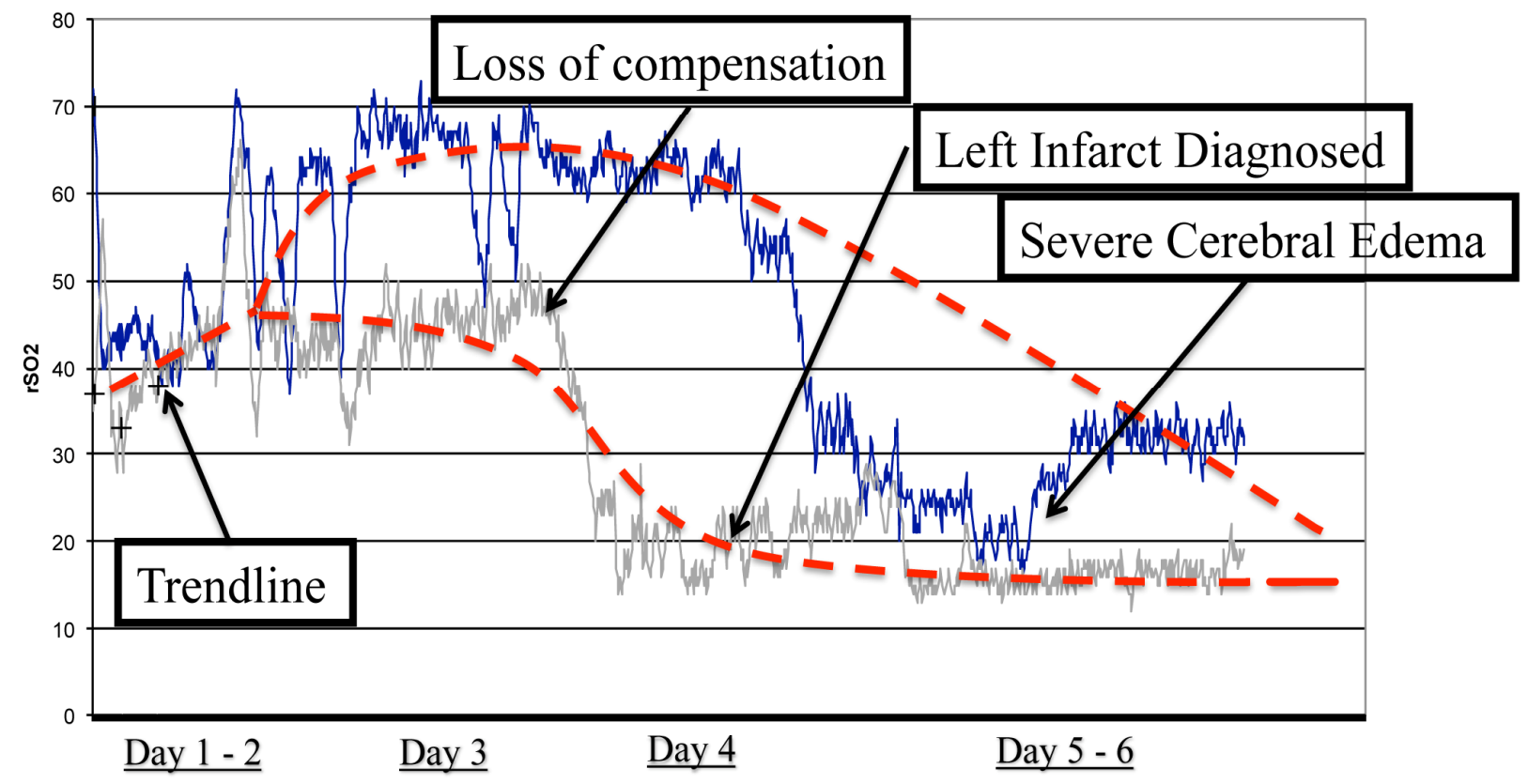


Figure 5. A lower limb event noted by NIRS in a patient initially without a distal perfusion cannula; 1700: Lower limb NIRS monitoring initiated. 1710: Right lower limb rSO2 tracing drop with normal left rSO2 tracings. 1747: Placement of a distal perfusion cannula in the right femoral artery improved right rSO2 to baseline. 1850: Distal perfusion cannula displacement with subsequent right rSO2 tracing drop. Replacement of cannula returns the tracings to baseline.

\section{Femoral Event}

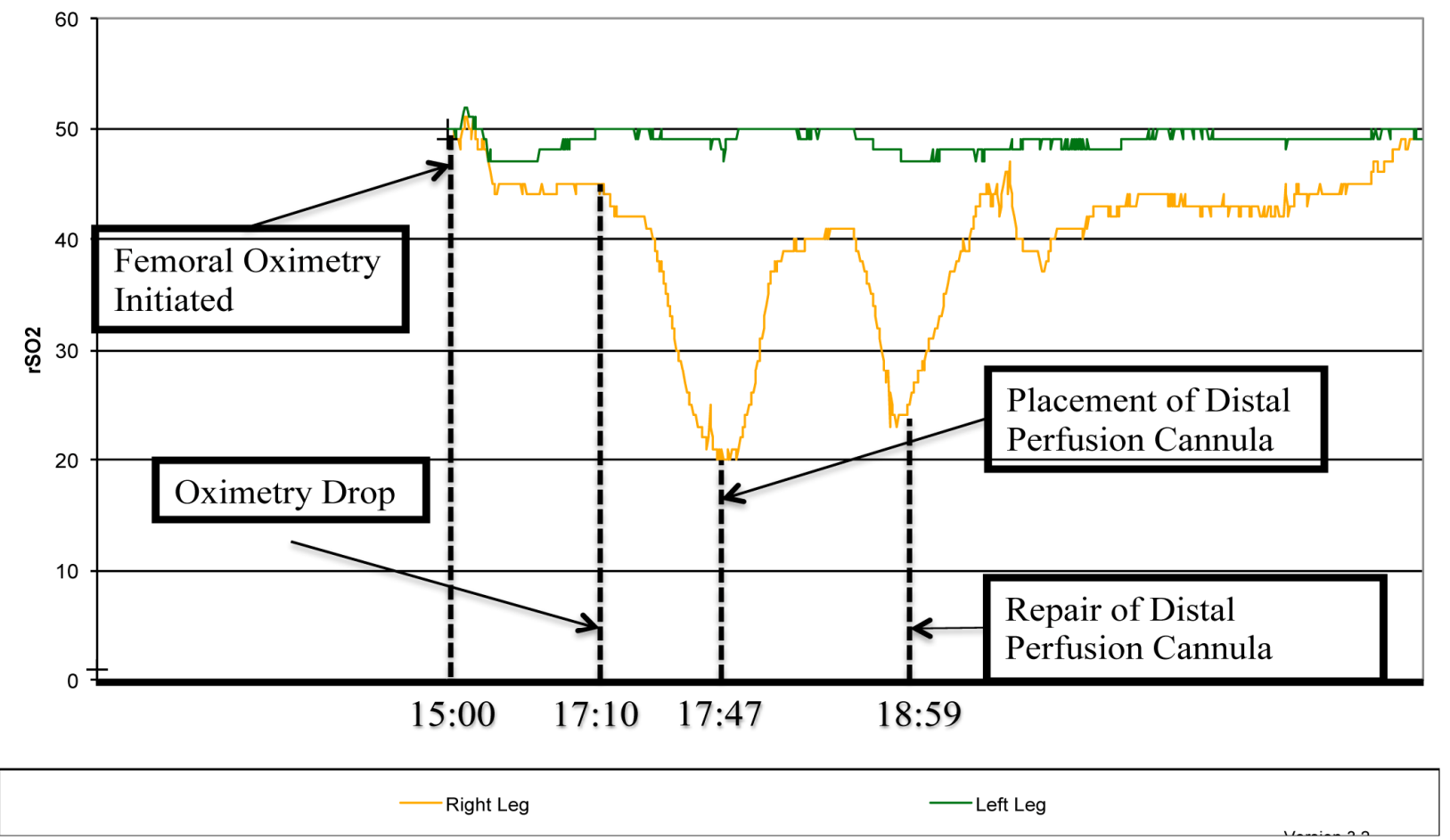


Figure 6. 6a (Left). Mixing of oxygenated and deoxygenated blood proximal to bifurcation of great vessels.

$6 \mathrm{~b}$ (Right). Mixing of oxygenated and deoxygenated blood distal to bifurcation of great vessels.

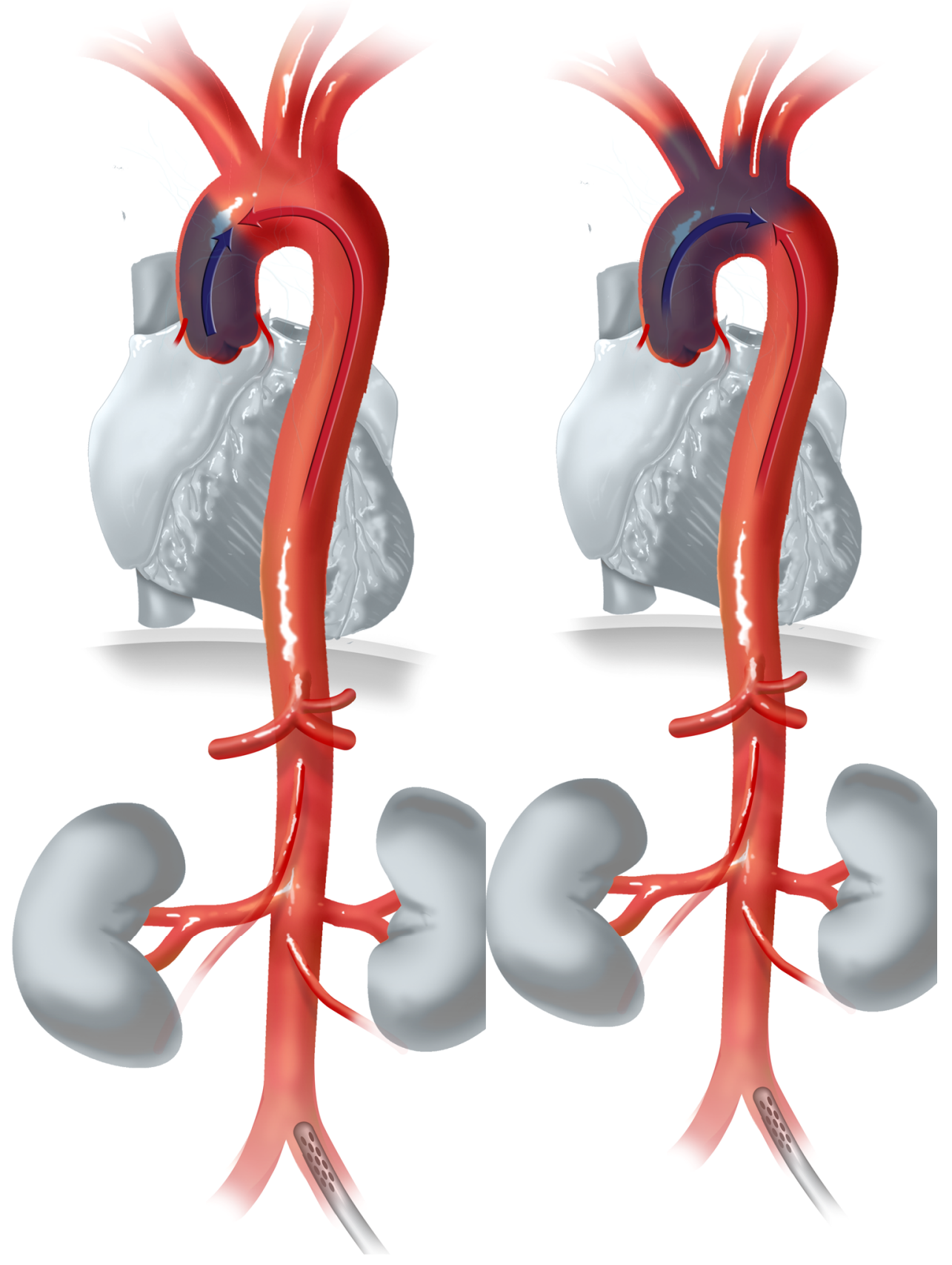




\section{REFERENCES}

1) Hill JD, O'Brien TG, Murray JJ, Dontigny L, Bramson ML, Osborn JJ, et al. Prolonged extracorporeal oxygenation for acute post-traumatic respiratory failure (shock-lung syndrome). Use of the Bramson membrane lung. N Engl J Med. 1972;286:629-34.

2) Mugford M, Elbourne D, Field D. Extracorporeal membrane oxygenation for severe respiratory failure in newborn infants. Cochrane Database Syst Rev. 2008;16:CD001340.

3) Zapol WM, Snider MT, Hill JD, Fallat RJ, Bartlett RH, Edmunds LH, et al. Extracorporeal membrane oxygenation in severe acute respiratory failure. A randomized prospective study. JAMA. 1979;242:2193-6.

4) Sidebotham D, McGeorge A, McGuinness S, Edwards M, Willcox T, Beca J. Extracorporeal membrane oxygenation for treating severe cardiac and respiratory disease in adults: Part 1-overview of extracorporeal membrane oxygenation. J Cardiothorac Vasc Anesth. 2009;23:886-92.

5) Sidebotham D, McGeorge A, McGuinness S, Edwards M, Willcox T, Beca J. Extracorporeal membrane oxygenation for treating severe cardiac and respiratory failure in adults: part 2technical considerations. J Cardiothorac Vasc Anesth. 2010;24:164-72.

6) Khoshbin E, Roberts N, Harvey C, Machin D, Killer H, Peek GJ, et al: Poly-methyl pentene oxygenators have improved gas exchange capability and reduced transfusion requirements in adult extracorporeal membrane oxygenation. ASAIO J. 2005;51:281-87.

7) Lawson DS, Ing R, Cheifetz IM, Walczak R, Craig D, Schulman S, et al: Hemolytic characteristics of three commercially available centrifugal blood pumps. Pediatr Crit Care Med. 2005;6:573-77.

8) Smedira NG, Moazami N, Golding CM, McCarthy PM, Apperson-Hansen C, Blackstone EH, et al. Clinical experience with 202 adults receiving extracorporeal membrane oxygenation for cardiac failure: survival at five years. J Thorac Cardiovasc Surg. 2001;122:92-102.

9) Hei F, Lou S, Li J, Yu K, Liu J, Feng Z, et al. Five-year results of 121 consecutive patients treated with extracorporeal membrane oxygenation at Fu Wai Hospital. Artif Organs. 2011;35:572-8.

10) Brodie D, Bacchetta M. Extracorporeal membrane oxygenation for ARDS in adults. N Engl J Med. 2011;365:1905-14.

11) Goldman S, Sutter F, Ferdinand F, Trace C. Optimizing intra-operative cerebral oxygen delivery using noninvasive cerebral oximetry decreases the incidence of stroke for cardiac surgical patients. Heart Surg Forum. 2004;7:E376-81.

12) Murkin JM, Adams SJ, Novick RJ, Quantz M, Bainbridge D, Iglesias I. Monitoring brain oxygen saturation during coronary bypass surgery: a randomized, prospective study. Anesth Analg. 2007;104:51-8.

13) Jöbsis FF. Noninvasive, infrared monitoring of cerebral and myocardial oxygen sufficiency and circulatory parameters. Science. 1977;198:1264-7.

14) Ferrari M, Giannini I, Sideri G, Zanette E. Continuous non invasive monitoring of human brain by near infrared spectroscopy. Adv Exp Med Biol. 1985;191:873-82.

15) Samra SK, Dy EA, Welch K, Dorje P, Zelenock GB, Stanley JC. Evaluation of a cerebral oximeter as a monitor of cerebral ischemia during carotid endarterectomy. Anesthesiology. 2000;93(4):964-70. 
16) Ali AM, Green D, Zayed H, Halawa M, El-Sakka K, Rashid HI. Cerebral monitoring in patients undergoing carotid endarterectomy using a triple assessment technique. Interact Cardiovasc Thorac Surg. 2011;12(3):454-7.

17) Papademetriou MD, Tachtsidis I, Leung TS, Elliott MJ, Hoskote A, Elwell CE. Cerebral and peripheral tissue oxygenation in children supported on ECMO for cardio-respiratory failure. Adv Exp Med Biol. 2010;662: 447-53.

1 18) Rais-Bahrami K, Rivera O, Short BL. Validation of a noninvasive neonatal optical cerebral oximeter in veno-venous ECMO patients with a cephalad catheter. J Perinatol. 2006;26:62835 .

19) Mateen FJ, Muralidharan R, Shinohara RT, Parisi JE, Schears GJ, Wijdicks EF. Neurological Injury in Adults Treated With Extracorporeal Membrane Oxygenation. Arch Neurol. 2011 Aug 8

| 20) Lan C, Tsai PR, Chen YS, Ko WJ. Prognostic factors for adult patients receiving extracorporeal membrane oxygenation as mechanical circulatory support: a 14- year experience at a medical center. Artif Organs. 2010;34:E59-E64.

| 21) Ko WJ, Lin CY, Chen RJ, Wang SS, Lin FY, Chen YS. Extracorporeal membrane oxygenation support for adult postcardiotomy cardiogenic shock. Ann Thorac Surg. 2002;73:538-45.

22) Foley PJ, Morris RJ, Woo EY, Acker MA, Wang GJ, Fairman RM, et al. Limb ischemia during femoral cannulation for cardiopulmonary support. J Vasc Surg. 2010;52:850-3.

23) Arbabi S, Brundage SI, Gentilello LM. Near-infrared spectroscopy: a potential method for continuous, transcutaneous monitoring for compartmental syndrome in critically injured patients. J Trauma. 1999;47(5):829-33

24) Gentilello LM, Sanzone A, Wang L, Liu PY, Robinson L. Near-infrared spectroscopy versus compartment pressure for the diagnosis of lower extremity compartmental syndrome using electromyography-determined measurements of neuromuscular function. J Trauma. $\underline{2001 ; 51(1): 1-8}$ 